\title{
Dying sweetly: pregnancy, diabetes, and CoVID-19
}

\section{Opinion}

An unseen menace has stealthily made its way into our world. It has left in its stead a dissolution of all that we hold dear-taken lives, impacted our liberty, and surely stolen our happiness. The big disease with the tiny name, CoVID-19, is caused by a corona virus, of which four types exist - alpha, beta, delta, and gamma. CoVID-19 is a beta coronavirus, the difference between the four highlighted is noted only by changes in their protein composition. These viruses are globally distributed and impact both humans and animals. While the majority of human infections are mild, two distant relatives of COVID-19, SERS (Severe Acute Respiratory Syndrome) and MERS (Middle East Respiratory Syndrome) have been responsible for significant outbreaks of lethal pneumonias. Although the overall mortality rate of CoVID-19 appears to be lower in comparison to the other two, rates of infectivity are higher; additionally patients with co-morbidities are likely to manifest more severe disease and subsequently higher mortality.

For the women who are pregnant in the time of CoVID-19, joy and anticipation has been replaced with worry, anxiety, and trepidation. Questions abound concerning fetal developmental sequelae, if vertical transmission in utero or during delivery occurs, or if the virus is excreted into breast milk-potentially infecting the neonatequestions which no health provider has a DEFINITIVE answer to. For women with high risk conditions such as Lupus, hypertension, or diabetes, the dread is palpable. Why? Because it has been noted across all media that individuals with co-morbid conditions have poor outcomes if infected with CoVID-19. To this circumstance, there is abject proof, particularly for diabetics. Diabetes appears to be the bait that attracts this virus to a host, like a moth to a flame.

From the paucity of available studies gleaned from China, it has been shown that diabetes mellitus, as a distinctive co-morbidity, is associated with more severe disease, acute respiratory distress syndrome, and increased mortality. Pregnancy in and of itself confers a circumstance of vulnerability for women.

a. The immunologic status of the pregnant woman is depressed. While any foreign organism initiates the body's defense system to attack, in pregnancy the fetus IS the foreign organism and the feto-placental unit produces chemicals, which in effect, depresses the mothers immune system - this allowing the fetus to maintain its existence in the womb, and to grow and thrive unharmed.

b. The growth of the uterus displaces the lungs upward in the body, thus comprising a change in both their function and capacity.

c. Hormones from the placenta induce a diabetic state (hence the rationale for screening women for diabetes during pregnancy)

d. Pregnancy additionally increases the propensity for abnormal clotting to occur. COVID-19 appears to induce abnormal clotting - thus promoting an additive effect in pregnancy.

Diabetes effects on the body, particularly if poorly controlled, are many - but the main derangement reflects an increase in inflammation/
Volume 6 Issue 3 - 2020

\author{
Kecia Gaither \\ Perinatal Services/Maternal Fetal Medicine, USA
}

Correspondence: Kecia Gaither, Director, Perinatal Services/ Maternal Fetal Medicine, NYC Health+ Hospitals/Lincoln 234 East I49 th Street, Bronx, NY I045I, USA, Tel (914) 610-0425, Email keciagaithermd@aol.com

Received: April 24, 2020 | Published: June 01, 2020

oxidative stress, a decrease in immunological competency, and a derangement of clotting factors, increasing the risk of clot formation in the body - now a triple additive effect. This pathological conditionpoorly controlled diabetes, coupled with the normal physiological changes of pregnancy, make this particular cohort of patients one that extreme oversight need be paid if CoVID-19 infection occurs.

I am a Maternal Fetal Medicine specialist, and serve as the Director of Perinatal Services at NYC Health+Hospitals/Lincoln in the Bronx- located directly in one of the epicenters of Co-VID-19 infection in New York. My demographic is primarily underserved women of the African and Latin diaspora-for whom multiple comorbidities exist, particularly diabetes. In the last few months, I have noted, exclusively with my poorly controlled pregnant diabetics, exceedingly poor outcomes. This group has presented with stillbirths, diabetic ketoacidosis, cardiovascular dysfunction, respiratory distress and death. Why this group? Depressed immunity allowing for easier viral invasion? Potentiation of the inflammatory response already present by the virus? The absolute etiology has yet to be determined.

One piece of the Co-VID puzzle I am convinced that an inroad can be made with is via change in diet and nutritional supplementation to boost immunologic function. There are a number of studies out of China, Italy, and Korea that have utilized vitamin supplementationspecifically of Vitamins C and D for treating patients afflicted with CoVID-19. While these therapies are not a cure, their utilization appears to mitigate the severe effects of the infection. These vitamins appear to decrease inflammation/oxidative stress, inherent in CoVID-19 infection.

Vitamin C is a powerful antioxidant, and is involved in promoting the growth and spread of lymphocytes (those cells in the body which help fight infectious agents-like viruses and bacteria). Vitamin D is a fat soluble vitamin that is produced in the skin in conjunction with sunlight. For people of color, this vitamin is made in lower amounts in the body due to the effect of melanin - which inhibits the production. Vitamin D has a multitude of functions within the body, inclusive of maintaining cardiovascular health, stabilizing cognitive function/ mood, essential for bone health, and boosting immunity. Deficiency is correlated with the development of Type 2 diabetes, hypertension and preterm labor.

For pregnant women afflicted with diabetes, particularly women of color- who due to public health recommendations are remaining indoors, thus devoid of sunlight exposure, it may be prudent to 
supplement with both vitamins-but particularly Vitamin D. Changing dietary practices to include foods rich in both vitamins, in addition to foods known to naturally boost immunological function is advised. Onions, garlic, turmeric, citrus fruits, salmon, sardines, kale, collards, yellow/green/red peppers, and eggs are all wonderful choices-particularly in conjunction with prenatal vitamins containing Omega -3 .The medical community has a long way to go to find a cure for this disease - we are just at the beginning of a journey to even understand the pathophysiology and how it impacts men, women, children - pregnancy and lactation. In the interim, for pregnant diabetics especially, compliance with diet, insulin and/or oral medication for glycemic control are of utmost importance, in addition to hand washing, mask wearing, and maintaining social distancing. Natural augmentation of immune function with a healthy diet and vitamin supplementation with Vitamin $C$, and particularly vitamin $D$, will hopefully prove to be the armor to help protect pregnant diabetic women in the war against CoVID-19, and ultimately decrease the risk of them dying sweetly.

\section{Acknowledgments}

None.

\section{Conflicts of interest}

Author declare that there is no conflict of interest.

\section{Funding}

None. 